\title{
Exact Solutions for Power-Law Fluid of a Generalized Oldroyd-B Fluid with Oscillating Boundary Conditions
}

Y.Q. Liu, ${ }^{* * *}$ L.C. Zheng, * X.X. Zhang**

* Department of Mathematics and Mechanics, University of Science and Technology Beijing, Beijing 100083, China, ** Mechanical Engineering School, University of Science and Technology Beijing, Beijing 100083, China

Rheological constitutive equations with fractional derivatives [1] have been proved to be a valuable tool to handle viscoelastic properties. In general, the constitutive equations for generalized nonNewtonian fluids are modified from the well known fluid models by replacing the time derivative of an integer order with the so-called Riemann-Liouville fractional calculus operators [2].This paper consider the power-law fluid of an incompressible generalized Oldroyd-B fluid with oscillating boundary conditions.

The shear stress of power-law fluid of a generalized Oldroyd-B fluid is

$$
\left(1+\lambda \mathrm{D}_{t}^{\alpha}\right) \tau(y, t)=\left(1+\lambda_{r} \mathrm{D}_{t}^{\beta}\right) \kappa\left|\frac{\partial u(y, t)}{\partial y}\right|^{n-1} \frac{\partial u(y, t)}{\partial y},
$$

where $\tau(y, t)=S_{x y}(y, t)$ is the shear stress, $\quad v=\gamma|\partial u / \partial y|^{n-1}$ is the kinematic viscosity $(\kappa$ and $\gamma=\kappa / \rho$ are positive constant) [3]. The case $n=1$ corresponds to a Newtonian fluid, $0<n<1$ is "power law" relation proposed as being descriptive of pseudo-plastic non-Newtonian fluid while $n>1$ describes dilatant fluids. $\mathrm{D}_{t}^{\alpha}$ is the Riemann-Liouville fractional differentiation operator of order $\alpha$ with respect to $t$ defined as [1]. Let us consider an infinite plate at $y=0$ and incompressible power fluid which is in contact with the plate and occupies the region $y>0$. Initially the fluid as well as the plate is at rest, and at time $t=0^{+}$the plate oscillate in its plane with the velocity $V \cos (\omega t)$ or $V \sin (\omega t)(V$ is a constant), see FIG.1. The fluid is permeated by an imposed magnetic field; the magnetic body force is represented $\sigma B_{0}^{2} u$. In the absence of a pressure gradient in the $x$-direction, the motion equation and the associated initial and boundary conditions as follows:

$$
\left(1+\lambda \mathrm{D}_{t}^{\alpha}\right) \frac{\partial u(y, t)}{\partial t}=\left(1+\lambda_{r} \mathrm{D}_{t}^{\beta}\right) \frac{\partial}{\partial y}\left(\gamma\left|\frac{\partial u(y, t)}{\partial y}\right|^{n-1} \frac{\partial u(y, t)}{\partial y}\right)-\frac{\sigma B_{0}^{2}}{\rho}\left(1+\lambda \mathrm{D}_{t}^{\alpha}\right) u(y, t) .
$$

Initial condition:

$$
\begin{gathered}
u(y, 0)=\partial_{t} u(y, 0)=0, \quad y>0 . \\
u(0, t)=V \sin (\omega \mathrm{t}) \text { or } u(0, t)=V \cos (\omega \mathrm{t}), \quad t>0, \\
u(y, t), \quad \partial_{y} u(y, t) \rightarrow 0 \text { as } y \rightarrow \infty, \quad t>0 .
\end{gathered}
$$

The solutions for the velocity field and shear stress are obtained by using Taylor expansion method and the Fourier sine transform coupled with Laplace transform. The velocity profiles are displayed for different time $\omega t=k \pi / 4(k=1,2,3,4,5,6,7,8)$ with $\omega=1$ in FIG.2. FIG.3 shows the velocity profiles at different power-law index. The velocity is increasing with the increase of the power-law index, that is to say the velocity of dilatant fluids is greater than pseudo-plastic fluid [4].

\section{References}

[1] I.Podlubny, Fractional differential equations, New York: Academic Press, 1999.

[2] M. Khan et al., Math. Comput. Model., 49 (2009) 1519. 
[3] L. C. Zheng et al, J. Thermal Science, 13 (2004) 150.

[4] This research was supported by the National Natural Science Foundations of China (No.50936003, 51076012).

(a)

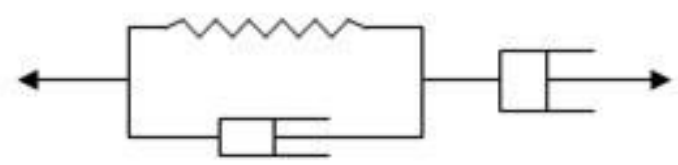

Oldroyd-B model

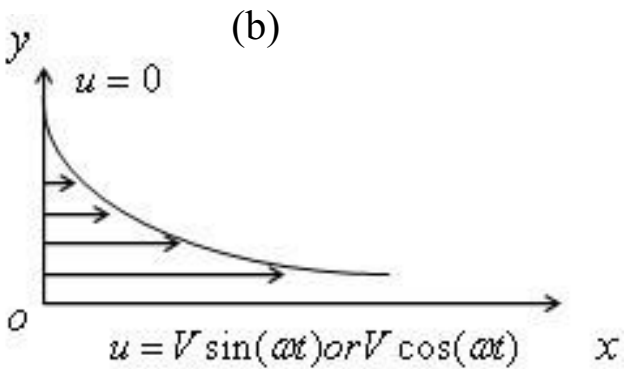

FIG. 1. (a) is the Oldroyd-B model, which is constituted by a spring and two dampers. The damping fluid is power-law fluid. (b) is the fluid flow model.

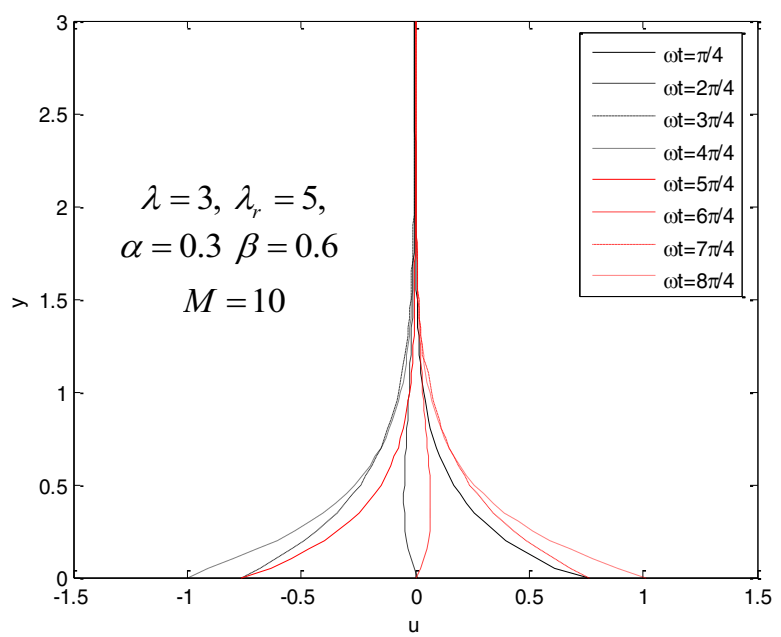

FIG. 2. Profiles of the velocity field at different times for $u(0, t)=V \cos (\omega \mathrm{t})$ with $\omega=1$.

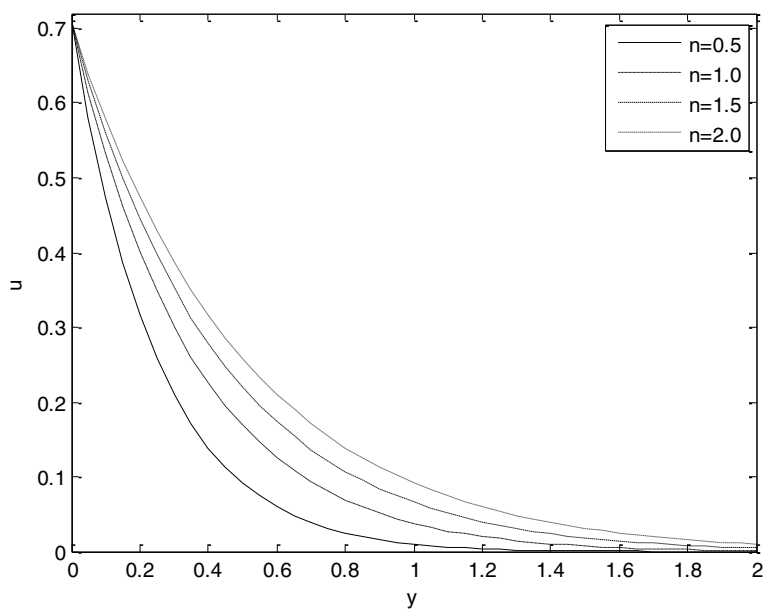

FIG. 3. The velocity profiles of the fluid with different power-law index $n$. 